\title{
Realidad virtual como buena práctica para trabajo en equipo con estudiantes de ingeniería
}

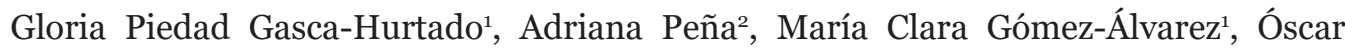 \\ Armando Plascencia-Osuna², Jose A. Calvo-Manzano ${ }^{3}$
}

gpgasca@udem.edu.co, adriana.pena@cucei.udg.mx, mcgomez@udem.edu.co,oskaarosunaa@ gmail.com,jacalvo@fi.upm.es

${ }^{1}$ Universidad de Medellín, Facultad de Ingeniería, Cra. 87 No. 30-65, Medellín, Antioquia, Colombia.

${ }^{2}$ Universidad de Guadalajara, CUCEI, Av. Revolución 1500, C.P. 44430, Guadalajara, Jalisco, México.

${ }^{3}$ Universidad Politécnica de Madrid, Facultad de Informática, Campus de Montegancedo, Boadilla del Monte, C.P. 28660 Madrid, España.

DOI: 10.17013/risti.16.76-91

Resumen: La realidad virtual es una alternativa de motivación de los estudiantes al darles la posibilidad de realizar acciones como volar u observar el entorno desde diferentes perspectivas de manera controlada. La interacción multiusuario en realidad virtual da lugar a los entornos virtuales colaborativos que favorecen el desarrollo de habilidades sociales como el trabajo en equipo, en particular en equipos con integrantes con ubicaciones geográficas diferentes. En estos contextos se encuentran diversas propuestas de aplicación de la realidad virtual que transportan a los estudiantes a entornos donde deben tomar decisiones conjuntas para lograr los objetivos propuestos. Sin embargo, estas propuestas no hacen énfasis en la importancia de reconocer las fortalezas o habilidades de cada miembro del equipo para mejorar su desempeño. El presente artículo propone un videojuego, basado en realidad virtual, que incorpora los principios de gamificación para promover y mejorar el trabajo en equipo en estudiantes de ingeniería.

Palabras-clave: realidad virtual; trabajo en equipo; gamificación; educación en ingeniería; entorno virtual colaborativo

\section{Virtual Reality as good practice for teamwork with engineering students}

Abstract: Virtual reality is an alternative for student's motivation, allowing them actions like flying or observing the environment from different perspectives in a controlled way. Multiuser interaction in virtual reality leads to collaborative virtual environments that promote the development of social skills in teamwork, in particular with members in different geographic locations. In these contexts there are different virtual reality application proposals transporting students to environments where they make team decisions for achieving intended goals. However, such proposals do not emphasize the importance of identifying the strengths of each team member for the improvement in the team performance. This paper presents a virtual reality-based videogame incorporating gamification principles for promoting and improving teamwork in engineering students. 
Keywords: virtual reality; teamwork, gamification; engineering education; collaborative virtual environment

\section{Introducción}

La Ingeniería es una disciplina basada en la ejecución de proyectos por parte de equipos de trabajo donde se requieren habilidades como negociación, comunicación efectiva y cooperación entre sus miembros (Platt, 2011). Este tipo de habilidades son difíciles de desarrollar a partir de una estrategia de enseñanza de carácter teórico. Por esta razón, vienen apareciendo diferentes iniciativas orientadas a la incorporación de los principios de los juegos (gamificación) en educación buscando generar mayor motivación y compromiso de los estudiantes frente a su proceso de aprendizaje (Lingard \& Barkataki, 2011). Una de estas iniciativas consiste en la utilización de realidad virtual para generar ambientes virtuales de colaboración donde los participantes trabajen de manera coordinada para el logro de los objetivos del equipo.

La realidad virtual en la enseñanza permite la simulación de entornos donde los participantes poseen características excepcionales sin riesgos para su seguridad ni la necesidad de realizar grandes inversiones ni que los participantes compartan la misma ubicación geográfica (Bricken, 1991). Existen varios estudios a gran escala (Di Blas \& Paolini, 2014) que evidencian los beneficios de esta tecnología para promover la colaboración. Sin embargo, en estos estudios no se tiene en cuenta de manera formal la importancia de identificar las fortalezas de cada miembro del equipo al momento de asignar funciones para mejorar su desempeño.

En el presente artículo se plantea un videojuego basado en realidad virtual para promover el trabajo en equipo en estudiantes de ingeniería aprovechando las ventajas de esta tecnología como: a) favorecer la interacción entre participantes con ubicaciones geográficas diversas y b) el nivel de realismo que se puede alcanzar con estos ambientes virtuales de colaboración.

La estructura del artículo es la siguiente: en la Sección 2 se describe el marco conceptual y los antecedentes que dan lugar al trabajo presentado; la Sección 3 presenta el diseño pedagógico y técnico del videojuego propuesto. Finalmente en la Sección 4 se plantean conclusiones y el trabajo futuro derivado del videojuego propuesto.

\section{Antecedentes}

El videojuego propuesto en este artículo se basa en cuatro pilares o ejes de trabajo: a) enseñanza de trabajo en equipo en estudiantes de ingeniería, b) incorporación de gamificación en educació, c) utilización de realidad virtual como estratregia de enseñanza y d) uso de realidad virtual para promover el trabajo en equipo. En la presente sección se describen cada uno de estos ejes.

\subsection{Enseñanza de trabajo en equipo en estudiantes de ingeniería}

Ingeniería es por naturaleza un proceso de colaboración, y la mayoría de los sistemas de producción están diseñados por equipos de trabajo durante largos períodos de tiempo. A pesar de que se reconoce el trabajo en equipo como parte de la naturaleza de 
la Ingeniería, a nivel industrial las empresas demandan habilidades de los profesionales asociadas al trabajo en equipo porque encuentran falencias relacionadas con dichas habilidades (Platt, 2011). Muchas de esas falencias son causadas porque en los programas universitarios pocas veces se tienen contenidos curriculares propios para la enseñanza de habilidades como el trabajo en equipo, debido a la dificultad que supone promover este tipo de habilidades en el estudiante.

Como forma de fomentar este tipo de habilidades en los estudiantes, en la mayoría de los casos, las universidades buscan mecanismos para desarrollar proyectos en el ejercicio de una clase de forma colaborativa y así motivar el trabajo en equipo. Sin embargo, estos esfuerzos son insuficientes para ayudar a los estudiantes a desarrollar o mejorar habilidades de trabajo en equipo de manera específica (Lingard \& Barkataki, 2011). Es por esto que la realidad virtual y un entorno virtual de colaboración pueden aportar un elemento diferenciador de apoyo para el mejoramiento de la enseñanza y la generación de habilidades de trabajo en equipo, de cooperación y de colaboración en los estudiantes.

\subsection{Gamificación en Educación}

La gamificación es una técnica para proponer dinámicas asociadas con el diseño de juegos en entornos en los que comúnmente no se aplican dichas dinámicas, con el fin de estimular y tener una interacción directa del usuario en el entorno (Lee \& Hammer, 2011). En educación, el uso de los conceptos y principios de gamificación permite disminuir las dificultades de enseñanza (Kiryajova, Angelova \& Yordakova, 2014). Con el fin de establecer metodologías de enseñanza y definir las áreas temáticas que responden a las necesidades de la industria del software, se generan propuestas (Honig. W.L., 2008), (Taran, 2007) y (Zapata, 2007) que buscan transformar el enfoque para enseñar los temas asociados a la mejora de procesos software, entre otros.

La gamificación se considera una oportunidad para optimizar la participación de los usuarios, implicar a los participantes de una forma más ágil en relación con la consecución de los logros e incrementar los indicadores de progreso de la mejora de procesos necesarios para el éxito de la implementación de buenas prácticas en las organizaciones (Dorling \& McCaffery, 2012).

La gamificación se constituye en una alternativa para utilizar los principios del juego en la enseñanza de ingeniería, en particular, ingeniería de software. Se han identificado casos de éxito en los que se logra un significativo incremento de la velocidad de aprendizaje y la asimilación de conceptos. Sin embargo, el principal alcance que se ha logrado con la incorporación de los principios de gamificación en ingeniería de software es motivar la fuerza de trabajo de los equipos que participan en el desarrollo de software, incluyendo las experiencias de implementación de gamificación en la industria.

El uso de la gamificación se ha extendido a la educación, tanto que existen organizaciones usando la gamificación para entrenar a sus empleados y en las universidades se utiliza como estrategia de enseñanza a los estudiantes, generación de ideas y nuevos conceptos.

IBM por ejemplo, ha creado una iniciativa llamada INNOVA8 para la enseñanza de conceptos complejos de gestión de proyectos para involucrar a los estudiantes en una compañía ficticia y generar una disciplina de responsabilidad frente a la toma de decisiones complejas (Kapp, 2012). 
En el área militar también existen ejemplos de la aplicación de los principios del juego enmarcados en la gamificación que dan cuenta de su uso en ambientes educativos (Kapp, 2012).

En el e-learning se identifican tendencias relacionadas con la gamificación, mediante la aplicación de la mecánica del juego y la dinámica de las tareas y procesos de e-learning es posible aumentar la participación de los usuarios con una aplicación de e-learning y sus tareas específicas.

Otras investigaciones que muestran la utilidad de gamificación en la educación son: Lee y Hammer (Lee \& Hammer, 2011) quienes explican el significado de gamificación, qué es y cómo se puede utilizar, al mismo tiempo que señalan las posibles desventajas en caso de mal uso. Los autores definen el uso de la mecánica del juego, dinámicas y marcos para promover comportamientos deseados. Fogg (Fogg, 2009) también indica que la gamificación puede ayudar a determinar ciertos comportamientos en las personas y así es posible identificar el uso de la gamification en el aprendizaje, y en e-learning (Muntean, 2011).

Aunque también existen investigaciones relacionadas con las desventajas y peligros que puede ofrecer el mal uso de la gamificación cuando no se ajusta a la finalidad de motivar a los estudiantes a participar y ofrecer apoyo a los docentes. En estos casos es posible que el resultado sea acostumbrar a los estudiantes a que deben aprender sólo cuando se proporciona una motivación extrínseca (Muntean, 2011).

Sin embargo, se identifica un aumento en el gamificación del aprendizaje y la enseñanza. Debido a elementos de juego tales como el tiempo, la precisión y los programas asociados a la formación que generan en el usuario una satisfacción que los anima a lograr los objetivos propuestos.

\subsection{Realidad virtual como estrategia de enseñanza}

La realidad virtual (VR por sus siglas en inglés) es un poderoso contexto motivacional en el aula y para los estudiantes (Chittaro \& Ranon, 2007), en el que se pueden controlar por ejemplo: el tiempo, el clima, las escalas y la física de los objetos. En ella, los participantes pueden tener habilidades como volar, ocupar un objeto como cuerpo virtual u observar el ambiente desde perspectivas que no serían posibles en el mundo real. Como ventajas relacionadas con la enseñanza están, entre otras: a) en un entorno virtual los materiales no se rompen o se gastan; b) se puede experimentar en forma segura; c) estar en localizaciones distantes o peligrosas; o ser parte del arte, música, teatro o literatura (Bricken, 1991). En este contexto, en 1999 Roussos et al. (1999) señalaron la importancia de investigar la eficacia educacional de la VR en situaciones específicas de aprendizaje y expandir los dominios. Así mismo, se propone desarrollar nuevas expresiones de eficacia educacional, dado que en la VR se pueden utilizar métodos de instrucción diferentes a la instrucción directa tradicional, que proporcionen a los estudiantes la oportunidad de desarrollar nociones más aceptadas científicamente (Eylon y Linn, 1988; Smith et al., 1993).

Por otro lado, el aprendizaje se beneficia con la interacción social (Vygotsky, 1978) yel trabajo en equipo porque mejora el desarrollo cognitivo así como el manejo de habilidades sociales (Chittaro \& Ranon, 2007). Cuando se trata de realidad virtual multiusuario, es decir un entorno virtual colaborativo (CVE por sus siglas en inglés), se distingue la participación 
síncrona con usuarios geográficamente distantes. Un CVE representa un medio de comunicación único, una tecnología preponderantemente visual que reúne personas y objetos remotos en una proximidad espacial que facilita la interacción (Wolff et al., 2008).

\subsection{Realidad virtual para enseñanza de trabajo en equipo}

Existe un gran número de proyectos educativos que utilizan los CVEs, conectando estudiantes con diferentes características y antecedentes académicos y culturales (e.g. Prasolova-Førland \& Divitini, 2003). Sin embargo, muchos menos en el ámbito de la educación formal y que sean utilizados durante la clase. Kennedy-Clark et al. (2009) presentan un estudio en el que estudiantes de historia utilizaron durante la clase un CVE. Éste los transportaba a Singapur en el siglo XIX donde hubo varias enfermedades epidémicas. En el estudio en aprendizaje basado en la investigación, Kennedy-Clark et al. (2009) concluyen que una estructura menos definida al inicio, ayuda a activar el conocimiento específico del dominio, así como a identificar ciertos conocimientos requeridos con lo que no se cuenta.

En una recopilación de varios estudios a gran escala, Di Blas \& Paolini (2014) presentan cuatro diferentes programas que tuvieron lugar entre el 2002 y el 2009, y que involucraron a más de 9.000 estudiantes con edades de entre 12 y 18 años, sobre el uso de CVEs para la educación formal. Entre sus conclusiones presentan varias lecciones aprendidas para promover la colaboración en esta tecnología, entre ellas, una muy importante en relación al trabajo aquí presentado, es la de dar oportunidad a la expresión de talentos diferentes.

En esta ocasión se pretende desarrollar un videojuego, basado en realidad virtual, enmarcado en una propuestas de enseñanza que incorpore los principios de gamificación para promover y mejorar el trabajo en equipo en estudiantes de ingeniería, considerando la VR como una oportunidad para mejorar la forma como se enseña ingeniería de software y garantizar la formación de los estudiantes en habilidades de trabajo en equipo.

\section{Videojuego para promover el trabajo en equipo en estudiantes de ingeniería}

El uso de la realidad virtual, además de tener un efecto motivacional (Bricken, 1991), genera en el usuario la sensación de estar en un lugar diferente de en el que realmente se encuentra, sensación de presencia. En una situación multiusuario, se espera que también genere una sensación de co-presencia, es decir, que lo haga sentir que otras personas están ahí, compartiendo la experiencia (Schroeder, 2002). Estas características hacen de la VR colaborativa una herramienta que fomenta que los participantes se involucren en la tarea que deben llevar a cabo. La interacción entre usuarios en un CVE es un valioso sustituto de una experiencia en la vida real, proporcionando a los participantes una práctica en primera persona que ayudará a generar un comportamiento espontáneo basado tanto en el involucramiento como en la sensación de co-presencia.

En un CVE se puede llevar a cabo una tarea espacial cuya logística en el mundo real sería mucho más complicada o incluso imposible. Aquí además, la atención de los usuarios es demandada para la manipulación de objetos, el monitoreo del escenario y las acciones de los demás. Esto fomenta una actitud mucho más enfocada en la tarea, en comparación con la vida real. Igualmente se considera que los escenarios son más simples que en la 
vida real ya que todos los objetos en él, están puestos por alguna razón relacionada con la tarea (Schroeder, 2011 p. 47).

El avatar del usuario, esto es, su representación gráfica en el mundo virtual, le permite interactuar con éste. En una situación multiusuario, el avatar es además el medio que permite a los usuarios estar conscientes de la presencia de los demás (Capin, Pandzic, Thalmann, \& Thalmann, 1997). Por otro lado, con el uso de un avatar, los participantes adquieren cierto anonimato. La apariencia de las personas se estandariza de alguna manera, lo que a su vez se espera afecte de igual forma el trato entre los participantes (Schroeder, 2011 p. 83)

Este conjunto de características deberá favorecer el conocimiento entre los miembros de un equipo respecto a la forma de trabajar colaborativamente, finalidad última de la aplicación que aquí se presenta.

\subsection{Principios de gamificación}

En respuesta a un estudio de necesidades identificadas sobre la formalización de la gamificación y necesidad de facilitar su adopción, Oprescu et al. proponen los siguientes principios de gamificación orientados a hacer más atractivo su uso (Oprescu, Jones, \& Katsikitis, 2014):

- Orientación: Los procesos gamificados permiten ubicar al usuario como centro de la experiencia.

- Elementos persuasivos: Los procesos gamificados incluyen elementos persuasivos basados en teorías psicológicas del sonido y conductuales.

- Orientación del aprendizaje: Enfocar el trabajo en la adquisición de conocimientos, el desarrollo de habilidades, los resultados de motivación o el cambio de comportamiento.

- Logro basado en recompensas: Enfocar el trabajo en un justificable y predecible retorno de la inversión.

- Adaptable generación Y: La llamada Generación Y es un tipo de población de rápido crecimiento de la fuerza de trabajo y buscan experiencias laborales que generen emociones, divertidas y atractivas.

- Factores de diversión: Incluir elementos de humor, el juego y la diversión como parte de los procesos del trabajo.

- Transformador: El uso balanceado y atractivo entre la colaboración y la competición con el fin de transformar los procesos de trabajo existentes en la organización.

- Bienestar orientado: Centrarse en el bienestar personal y organizacional.

- Generación de investigación: Los esfuerzos de investigación colaborativa deben ser motivados para conseguir inversión a futuro.

- Basado en el conocimiento: Basarse en el conocimiento ya sea como resultado o como retroalimentación.

Aunque los principios mencionados fueron propuestos para utilizarse en ambientes de trabajo gamificados, han sido considerados para analizar la productividad de dichos ambientes y para promover la salud en el lugar de trabajo. También son considerados como un buen punto de partida para otras áreas, en el caso de este trabajo los tomaremos como referente para la gamificación de un entorno de trabajo educativo. 
A continuación, se describe la propuesta de un videojuego utilizando los principios de gamificación para generar habilidades de trabajo en equipo y cooperación entre un grupo de estudiantes. Esta propuesta describe el diseño pedagógico del videojuego así como su diseño técnico como producto de software.

\subsection{Diseño pedagógico del videojuego}

Gómez Álvarez et al. (2014) proponen un método para diseñar instrumentos pedagógicos que parte de la identificación de la temática, propósito y características claves del instrumento hasta la evaluación inicial de instrumento con sesiones piloto para refinarlo antes de su aplicación al público objetivo. Este método fue aplicado para el diseño pedagógico del videojuego que se describe a continuación para la obtención de objetivos de aprendizaje, reglas y materiales requeridos para su aplicación.

\subsubsection{Objetivos de aprendizaje}

Al finalizar el videojuego el participante estará en capacidad de:

1. Identificar la importancia de definir un plan para el desarrollo de un proyecto. En este caso simulando la construcción de software utilizando la reconstrucción de un barco.

2. Reconocer la importancia de colaboración, teniendo en cuenta la necesidad de establecer acuerdos antes de la etapa de ejecución.

3. Mejorar el trabajo en equipo a partir de la especialización de funciones (selección de roles) a partir del reconocimiento de las limitaciones del entorno, así mismo pueden reconocer la importancia de la comunicación permanente entre los integrantes del equipo.

4. Reconocer la importancia de las características y fortalezas de cada miembro del equipo para la asignación de funciones específicas.

\subsubsection{Reglas}

Las reglas del videojuego fueron diseñadas para fomentar la colaboración entre cuatro participantes. En él, los participantes forman parte de una tripulación de un barco que después de una batalla quedó destruido y deben volver a armarlo. El esquema del barco y sus partes se muestra en la Figura 1. Los colores son importantes por las restricciones que maneja uno de los roles de los jugadores como se explica a continuación.

1. Los participantes visualizan las islas y las piezas del barco en cada isla. Tendrán la oportunidad de ver una descripción de los posibles roles a desempeñar junto con sus limitaciones físicas como se muestra en la tabla 1.

Las piezas para armar el barco están ubicadas en tres islotes diferentes de la siguiente forma (ver Figura 2):

Isla 1 - San Andrés: Piezas (1, 2, 3, 4, 9, 13, 16)

Isla 2 - Santa Catalina: Piezas $(5,6,7,8,12)$

Isla 3 - Santa Mónica: Piezas (10, 11, 14, 15) 


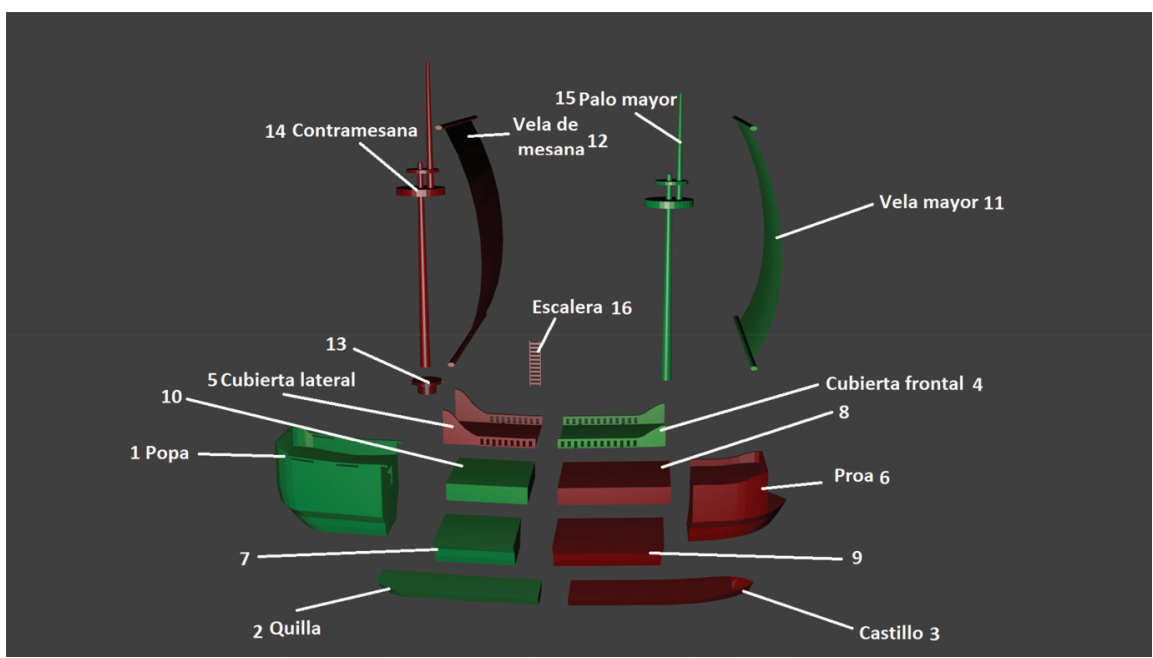

Figura 1 - Esquema del barco y sus partes.

\begin{tabular}{lll}
\hline Rol & Dificultad & Manejo en el sistema \\
\hline Tripulante 1 & $\begin{array}{l}\text { No tiene una } \\
\text { mano }\end{array}$ & $\begin{array}{l}\text { No se le permite cargar objetos pesados } \\
\text { (partes 1,6 de la Figura 1) }\end{array}$ \\
Tripulante 2 & $\begin{array}{l}\text { No tiene una } \\
\text { pierna }\end{array}$ & $\begin{array}{l}\text { Al navegar su avatar avanza más lentamente que el de } \\
\text { los demás (le toma el doble de tiempo trasladarse) }\end{array}$ \\
Tripulante 3 & Es mudo & $\begin{array}{l}\text { Su micrófono está desconectado, de tal forma que los } \\
\text { otros jugadores no escuchan su voz }\end{array}$ \\
Tripulante 4 & Es daltónico & $\begin{array}{l}\text { Para este jugador las piezas del barco aparecen todas } \\
\text { intercalarlas. }\end{array}$ \\
\hline
\end{tabular}

Tabla 1 - Roles y restricciones del videojuego

2. Los participantes tienen 5 minutos para seleccionar el rol que van a desempeñar en el video juego.

3. Los participantes pueden comunicarse vía voz para definir la estrategia a seguir en términos de: actividades a realizar (desplazamiento de piezas, armado), tripulante responsable y tiempo asignado.

4. Los participantes comienzan la construcción del barco en el videojuego con comunicación verbal y una vez hayan finalizado el barco podrán comparar el plan de la estrategia a seguir con las actividades reales realizadas (ejecución). 


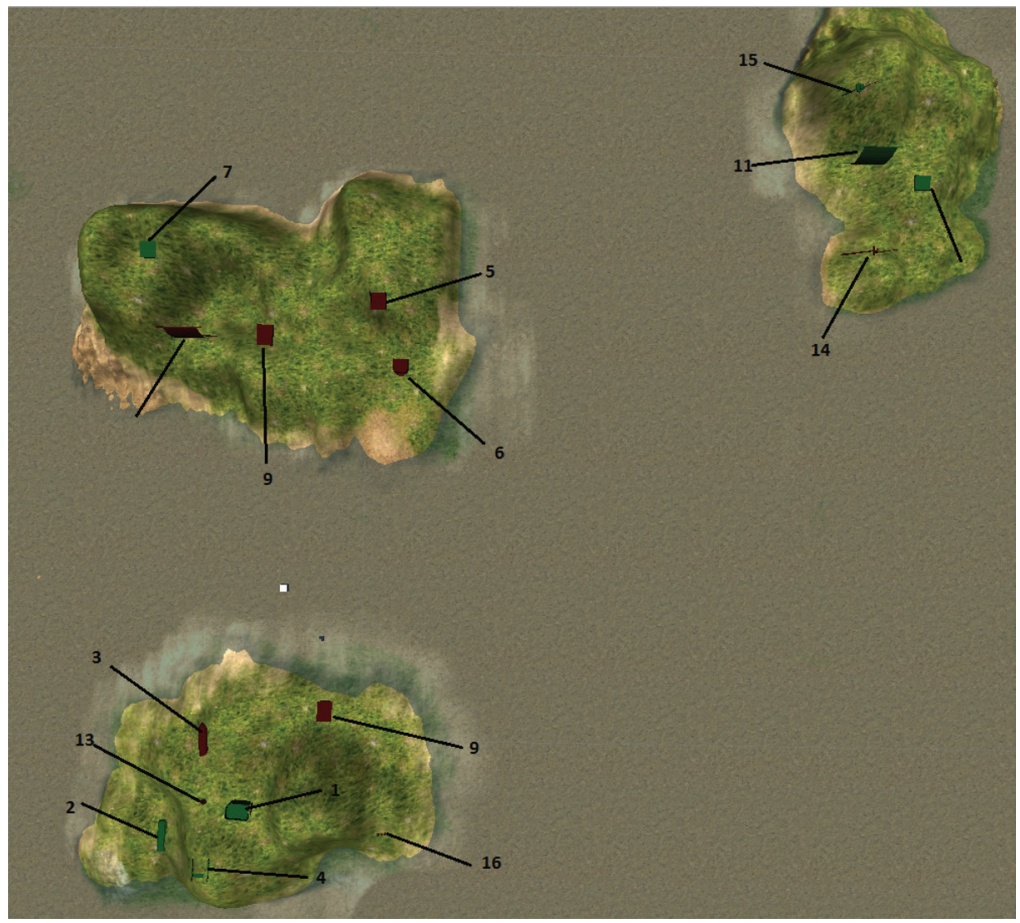

Figura 2 - Distribución de las piezas en las islas, al inicio del juego.

\subsubsection{Materiales}

El videojuego requiere:

- Un computador con acceso a internet, de tal manera que el participante pueda realizar una inmersión en el escenario que propone el juego.

- Un micrófono y audífono para poderse comunicar con otros miembros del equipo que tendrán ubicaciones geográficas diversas.

\subsection{Diseño técnico del juego}

Los requisitos del sistema obedecen a la descripción del videojuego. Entre los principales requisitos funcionales del sistema se encuentran:

- El sistema debe ser multiusuario y permitir la conexión vía Internet entre usuarios

- Cada participante deberá registrar un nombre que lo identificará. Esta identificación es válida tanto para el sistema como para los demás participantes.

- Se debe permitir la opción de levantar servidor así como conectarse a uno activo.

- La primera forma de comunicación será a través de un chat. Esto permitirá que queden registradas las especificaciones de la planeación. 
- Durante la fase de planeación estratégica, los usuarios tendrán acceso a las reglas del juego, la forma en que funciona el sistema virtual, el diagrama de cómo debe armarse el barco y el escenario virtual visto desde arriba para que identifiquen las islas y las partes del barco.

- Cada usuario deberá seleccionar un avatar que corresponde al rol que tendrá en el juego.

- En el escenario las acciones posibles son navegación, esto es su desplazamiento; selección y manipulación de objetos; y comunicación verbal.

- Las posiciones iniciales de los avatares se asignará aleatoriamente.

- La aplicación terminará en cierto tiempo.

De acuerdo con lo anterior, se define una arquitectura cliente-servidor (ver Figura 3). El primer jugador en conectarse debe levantar el servidor, de tal manera que en su computador se encontrarán tanto el cliente como el servidor, los jugadores que se conecten posteriormente se agregan a dicho servidor como clientes.

El juego fue desarrollado con el motor de gráficos Unity ${ }^{\mathrm{TM}}$ versión 4.3. Para la programación se utilizaron scripts tanto en lenguaje Java como en C\#. De tal manera que el paradigma de programación es basado en objetos a través de scripts, los mismos que son manejados directamente por Unity ${ }^{\mathrm{TM}}$. Cada script puede ser visto como una funcionalidad del sistema.

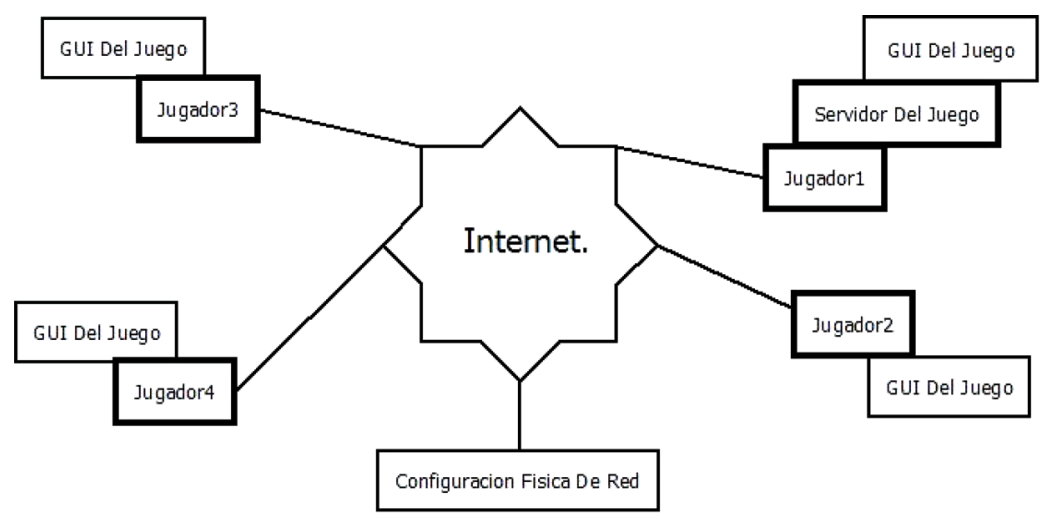

Figura 3-Arquitectura de la conexión

Los jugadores inician eligiendo un nombre de usuario (ver Figura 4). Luego tendrán que conectarse, eligiendo levantar el servidor o conectarse a uno existente (ver Figura 5). Una vez conectados se pueden comunicar a través de un chat, que les permite llegar a acuerdos sobre el plan de acción.

Posteriormente, cada jugador selecciona un avatar humanoide de acuerdo al rol que tendrá en el juego, que hará las veces de su representación gráfica en el juego (ver Figura 6). 


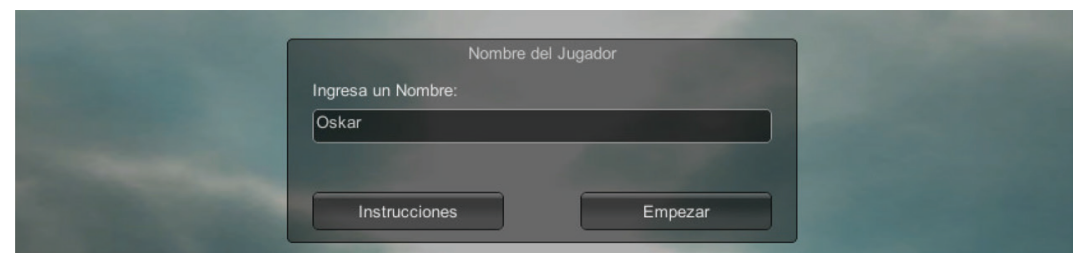

Figura 4 - Pantalla de inicio del videojuego

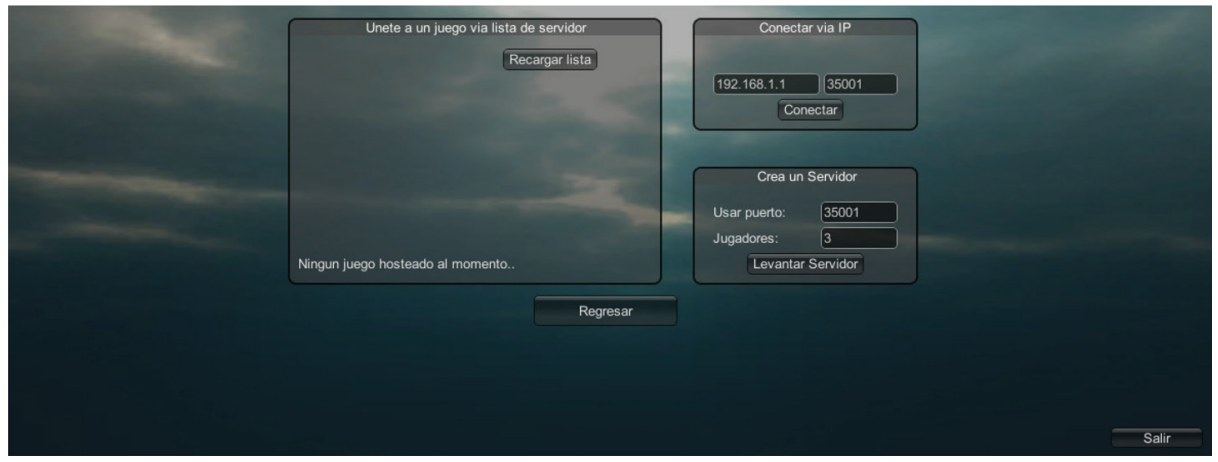

Figura 5 - Pantalla para levantar servidor o conectarse a uno existente.

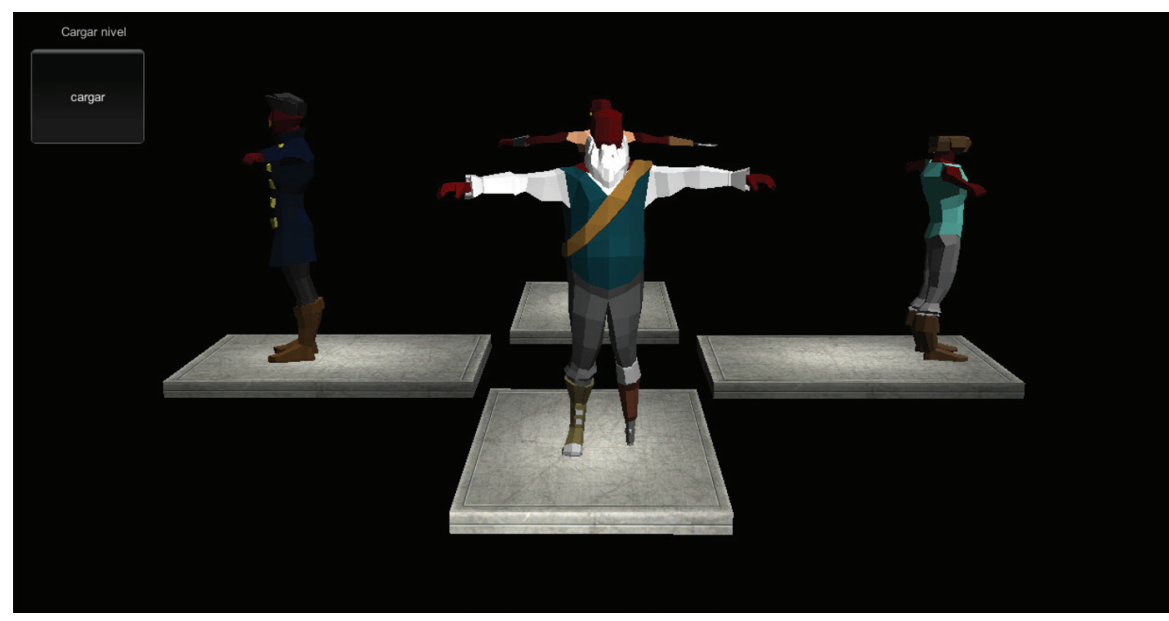

Figura 6 - Plataforma rotatoria para la selección del avatar

Cuando se han seleccionado los avatares se inicia el escenario virtual en el que se llevará a cabo la tarea, como se muestra en la Figura 7. Esta secuencia está representada en el Diagrama de Estados de la Figura 8. 


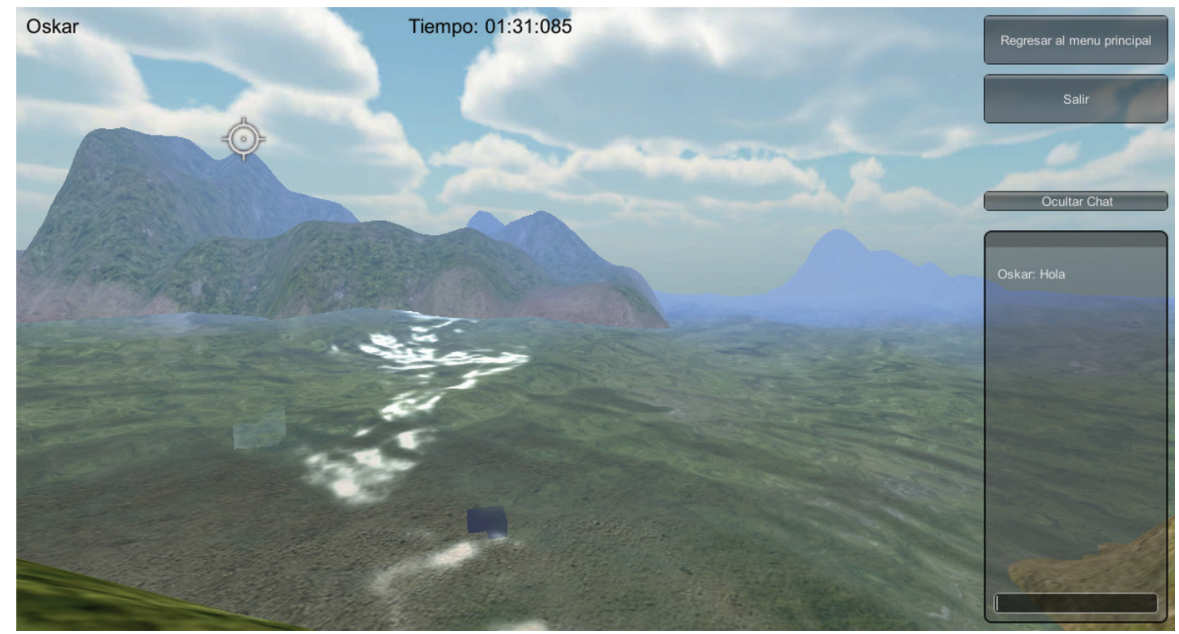

Figura 7 - Escenario virtual visto desde la posición de uno de los jugadores.

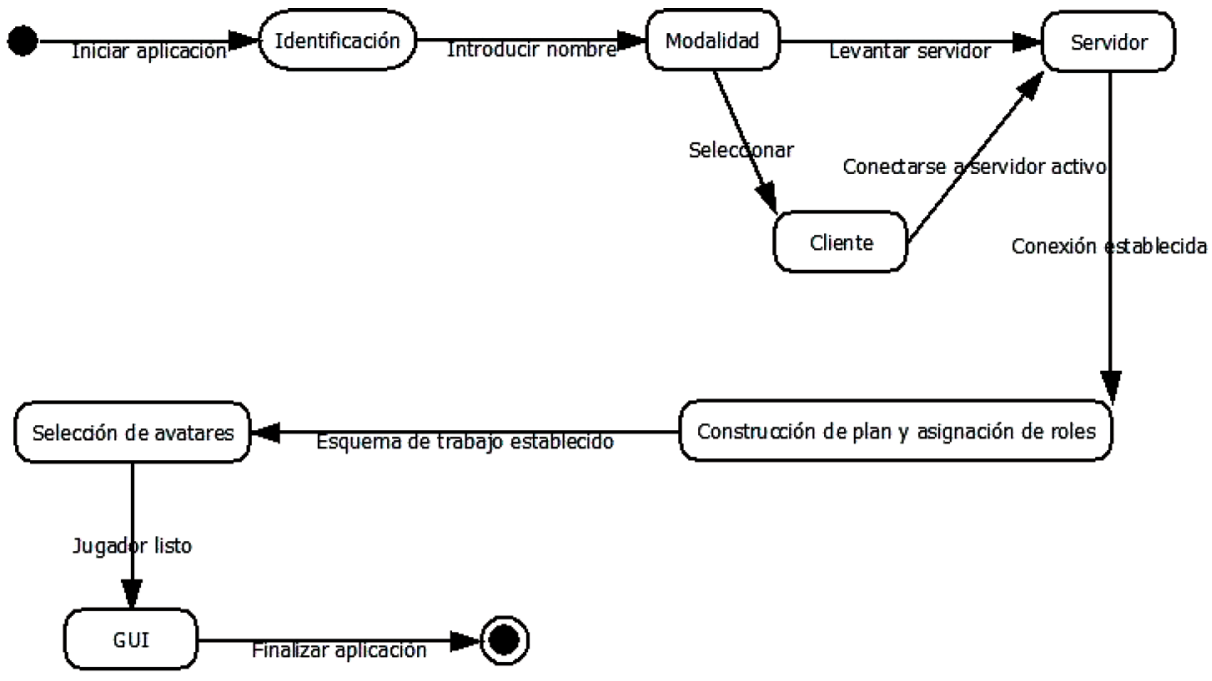

Figura 8 - Diagrama de estados para la conexión al mundo virtual

El avatar de los usuarios está en primera persona, esto significa que ellos no pueden verse a sí mismos, tal y como sucede en la vida real, sólo pueden ver sus extremidades (brazos y piernas). Para facilitar la identificación, el nombre de usuario se despliega en la parte superior izquierda del avatar.

En el escenario los usuarios pueden llevar a cabo diferentes acciones: seleccionar objetos, manipular objetos seleccionados (mover o rotar), navegar y hablar. Cada una de estas acciones se comunica al servidor mediante un RPC (remote procedure call). Los RPCs a su vez son enviados a todos los clientes para actualizar su escenario conforme 


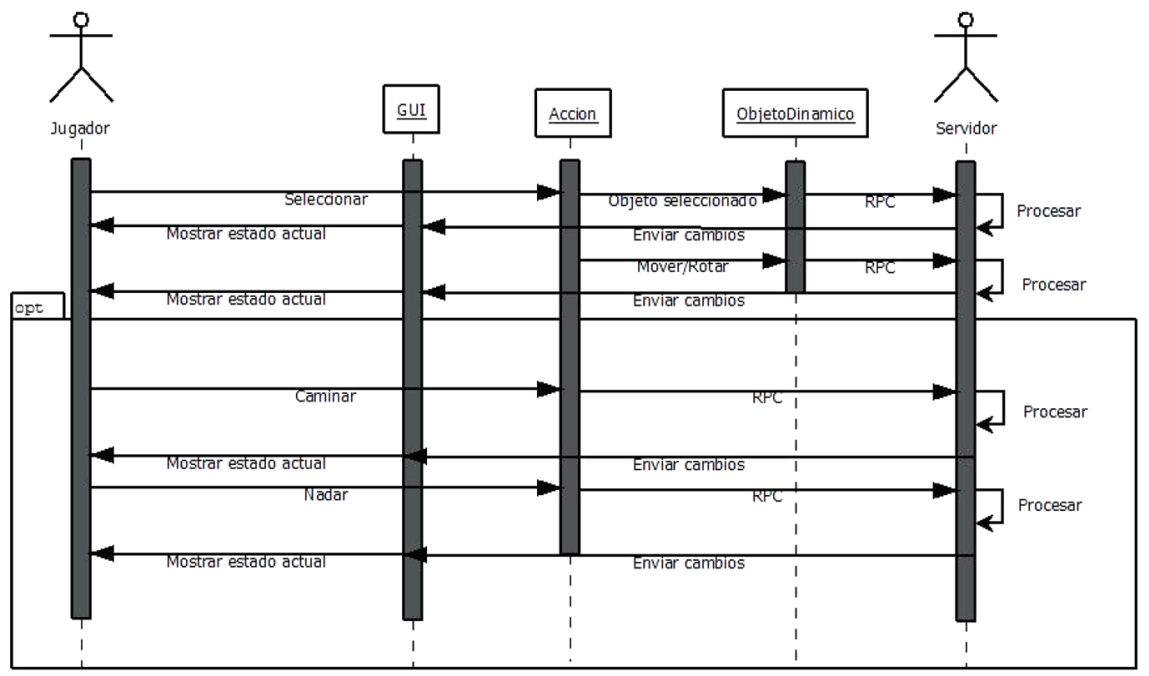

Figura 9 - Diagrama de Secuencia de las acciones del jugador.

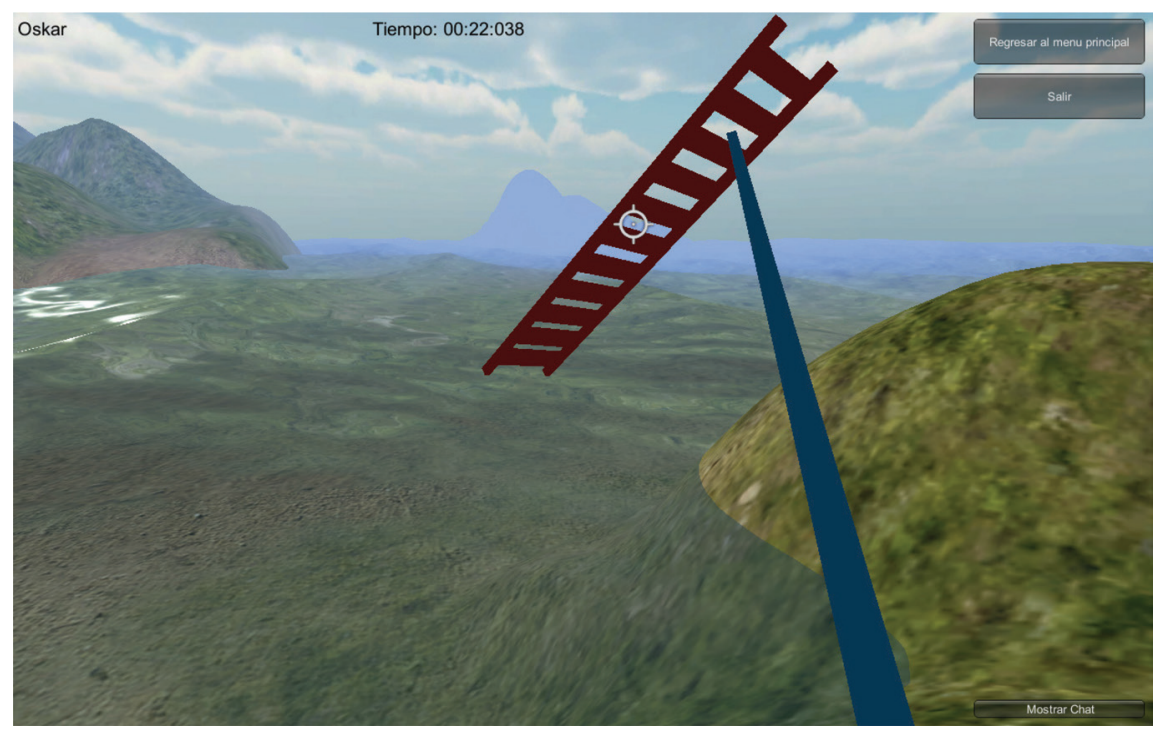

Figura 10 - Selección y movimiento de una parte del barco

a las acciones que cada cliente haya realizado, esto puede observarse en la gráfica de secuencia UML que se muestra en la Figura 9.

Para la selección de objetos, se dibuja una línea de la mano del avatar hasta el objeto seleccionado (técnica ray-casting). Esto permite tanto, al usuario como a los demás 
participantes, saber cuándo alguien ha tomado un objeto (ver Figura 10). Los dos tipos de navegación de los avatares son: a) caminando, es decir, cuando el usuario está en tierra y b) nadando, es decir cuando el usuario está en el agua. Ambos se ejecutan dando dirección con el ratón (mouse) y las flechas.

\section{Conclusiones y trabajo futuro}

La realidad virtual es una tecnología que favorece el trabajo colaborativo en los equipos y que permite a cada uno de los participantes involucrarse con las tareas asignadas para el logro de los objetivos comunes. De esta forma los entornos virtuales colaborativos permiten identificar patrones de comportamiento delos participantesylaimplementación de buenas prácticas para favorecer el trabajo colectivo así como identificar aciertos y fallas al momento de establecer la estrategia para resolver problemas específicos dentro de este entorno.

Entre las ventajas de la utilización de un videojuego basado en VR para fomentar el trabajo en equipo en estudiantes de ingeniería se pueden resaltar:

- Control del tiempo y de los materiales que utilizarán los participantes durante el juego.

- El participante puede sentirse parte del ambiente al observarlo en dos dimensiones.

- Costos bajos para la generación de nuevas versiones de videojuego, ya que sólo debe generarse la nueva versión del programa y no es necesario incurrir en compra de materiales consumibles.

- Simular las características y limitaciones de los participantes que no tienen en la vida real.

- Facilitar la interacción entre miembros de un equipo distribuido geográficamente mediado por las TIC y la realidad virtual.

El videojuego propuesto fue implementado siguiendo los principios de gamificación requeridos para combinar motivación y aprendizaje en la estrategia de enseñanza. Particularmente este videojuego busca promover el trabajo en equipo en estudiantes de ingeniería haciendo énfasis en la importancia de identificar las fortalezas de cada miembro del equipo para aportar al logro de los objetivos propuestos (especialización de funciones).

Como líneas de trabajo futuro se plantean:

1. Aplicar el videojuego propuesto en asignaturas de primeros semestres de ingeniería como alternativa para la formación de equipos de trabajo.

2. Complementar el videojuego con una estrategia de evaluación que permita conocer la percepción de los estudiantes frente al videojuego y a la estrategia de enseñanza basada en VR en contraste con las estrategias de enseñanza tradicionales como clases magistrales o estudios de caso. 


\section{Referencias}

Bricken, M. (1991). Virtual reality learning environments: Potentials and challenges. Computer Graphics, 25(3), 178-184. doi: http://dx.doi.org/10.1145/126640.126657.

Capin, T. K., Pandzic, I. S., Thalmann, N. M., \& Thalmann, D. (1997). Realistic avatars and autonomous virtual humans in VLNET networked virtual environments. From Desktop to Webtop: Virtual Environments on the Internet, WWW and Networks, International Conference, Bradford, UK.

Chittaro, L., \& Ranon, R. (2007). Web3D technologies in learning, education and training: Motivations, issues, opportunities. Computers \& Education Journal, 49(2), 3 -18. doi: http://dx.doi.org/10.1016/j.compedu.2005.06.002.

Di Blas, N., \& Paolini, P. (2014). Multi-User Virtual Environments Fostering Collaboration in Formal Education. Educational Technology \& Society, 17(1), 54-69.

Dorling, A., \& McCaffery, F. (2012). The Gamification of SPICE. In A. Mas, A. Mesquida, T. Rout, R. O'Connor \& A. Dorling (Eds.), Software Process Improvement and Capability Determination (Vol. 290, pp. 295-301): Springer Berlin Heidelberg. doi: http://dx.doi.org/10.1007/978-3-642-30439-2_35.

Fogg, B. J. (2009). A behavior model for persuasive design. Paper presented at the Proceedings of the 4th international Conference on Persuasive Technology. doi: http://dx.doi.org/10.1145/1541948.1541999.

Gómez Álvarez, M. C., Piedad Gasca-Hurtado, G., Villalón, C. M., Antonio, J., \& San Feliu Guilabert, T. (2014, June). Design of a pedagogic instrument for teaching software process improvement: Teaching instrument for university and business environments. In Information Systems and Technologies (CISTI), 2014 9th Iberian Conference on (pp. 1-7). IEEE. doi: http://dx.doi.org/10.1109/CISTI.2014.6876873.

Honig. W.L. (2008). Teaching Successful "Real-World" Software Engineering to the "Net" Generation: Process and Quality Win! . Paper presented at the CSEET 'o8. IEEE 21st Conference onSoftware Engineering Education and Training. doi: http://dx.doi.org/10.1109/CSEET.2008.38.

Kapp, K. M. (2012). The gamification of learning and instruction: game-based methods and strategies for training and education: John Wiley \& Sons. doi: http://dx.doi.org/10.4018/jgcms.2012100106.

Kennedy-Clark, S., Jacobson, M., \& Reimann, P. (2009) Productive Failure in Inquiry Learning in a Multi-User Virtual Environment. Proceedings of Ann. Conf. Australasian Soc. for Computers in Learning in Tertiary Education (ASCILITE), pp. 524-527.

Kiryakova, G., Angelova, N., \& Yordanova, L. (2014). Gamification in education. Proceedings of 9th International Balkan Education and Science Conference.

Lee, J. J., \& Hammer, J. (2011). Gamification in education: What, how, why bother? Academic Exchange Quarterly, 15(2), 146. 
Lingard, R., \& Barkataki, S. (2011, 12-15 Oct. 2011). Teaching teamwork in engineering and computer science. Paper presented at the Frontiers in Education Conference (FIE), 2011. doi: http://dx.doi.org/10.1109/FIE.2011.6143000.

Muntean, C. I. (2011). Raising engagement in e-learning through gamification. Paper presented at the Proc. 6th International Conference on Virtual Learning ICVL.

Oprescu, F., Jones, C., \& Katsikitis, M. (2014). I PLAY AT WORK-ten principles for transforming work processes through gamification. Frontiers in psychology, 5. doi: http://dx.doi.org/10.3389/fpsyg.2014.00014.

Platt, J. R. (2011). Career Focus: Software Engineering. IEEE-USA Today's Engineer Online.

Prasolova-Førland, E., \& Divitini, M. (2003). Collaborative virtual environments for supporting learning communities: an experience of use. Paper presented at the 12th International Conference on Supporting Group Work, USA, 58-67. doi: http://doi.acm.org/10.1145/958160.958170.

Roussos, M., Johnson, A., Moher, T., Leigh, J., Vasilakis, C., \& Barnes, C. (1999). Learning and building together in an immersive virtual world. Presence: Teleoperators and Virtual Environments, 8(3), 247-263. doi: http://dx.doi.org/10.1162/105474699566215.

Schroeder, R. (2011). In Kirlik A. (Ed.), Being there together: Social interaction in shared virtual environments. New York: Oxford University Press.

Taran, G. (2007). Using Games in Software Engineering Education to Teach Risk Management. Paper presented at the Proceedings of the 2oth Conference on Software Engineering Education \& Training, Dubin, Ireland. doi: http://dx.doi.org/10.1109/CSEET.2007.54.

Vygotsky, L. S. (1978). Mind in society: The development of higher psychological processes. Cambridge, MA: Harvard University Press.

Wolff, R., Roberts, D., Murgia, A., Murray, N., Rae, J., Steptoe, W., et al. (2008). Communicating eye gaze across a distance without rooting participants to the spot. Distributed Simulation and Real-Time Applications, 2008. DS-RT 2008. 12th IEEE International Symposium, Vancouver, British Columbia, Canada. doi: http://dx.doi.org/10.1109/DS-RT.2008.28.

Zapata, C.y.A., G. (2007). Requirements Game: Teaching Software Projects Management. CLEI Electronic Journal, 1. doi: http://www.clei.org/cleiej/paper.php?id=133. 